\title{
Risk factors for mortality among neonates admitted to a special care unit in a low- resource setting
}

Francesco Cavallin ${ }^{1}$, Teresa Bonasia ${ }^{2,3}$, Desalegn Abebe Yimer ${ }^{4}$, Fabio Manenti ${ }^{2,5}$, Giovanni Putoto ${ }^{5}$ and Daniele Trevisanuto ${ }^{6^{*}}$ (D)

\begin{abstract}
Background: Although under-5 mortality has decreased in the last two decades, neonatal mortality remains a global health challenge. Despite achieving notable progress, Ethiopia has still one of the highest neonatal mortality rates worldwide. We aimed to assess the risk factors for mortality among neonates admitted to a special care unit in a referral hospital in rural Ethiopia.

Methods: This was a retrospective observational study including all 4182 neonates admitted to the special care unit of the St. Luke Wolisso Hospital (Ethiopia) from January 2014 to December 2017. Data were retrieved from hospital charts and entered in an anonymized dataset. A logistic regression model was applied to identify predictors of mortality and effect sizes were expressed as odds ratios with 95\% confidence intervals.
\end{abstract}

Results: Proportion of deaths was 17\% (709/4182 neonates). Neonates referred from other health facilities or home (odds ratio $1.52,95 \%$ confidence interval 1.21 to 1.91), moderate hypothermia at admission (odds ratio 1.53, 95\% confidence interval 1.09 to 2.15) and diagnosis of late-onset sepsis (odds ratio 1.63, 95\% confidence interval 1.12 to 2.36), low birthweight (odds ratio 2.48, 95\% confidence interval 2.00 to 3.09), very low birthweight (odds ratio 11.71, 95\% confidence interval 8.63 to 15.94), extremely low birthweight (odds ratio 76.04, 95\% confidence interval 28.54 to 263.82), intrapartum-related complications (odds ratio 4.69, 95\% confidence interval 3.55 to 6.20 ), meconium aspiration syndrome (odds ratio 2.34, 95\% confidence interval 1.15 to 4.43), respiratory distress (odds ratio 2.25, 95\% confidence interval 1.72 to 2.95 ), other infections (odds ratio 1.92, 95\% confidence interval 1.31 to 2.81 ) or malformations (odds ratio $2.32,95 \%$ confidence interval 1.49 to 3.57 ) were associated with increased mortality. Being admitted in 2017 vs. 2014 (odds ratio 0.71, 95\% confidence interval 0.52 to 0.97), and older age at admission (odds ratio $0.95,95 \%$ confidence interval 0.93 to 0.97 ) were associated with decreased likelihood of mortality.

Conclusions: The majority of neonatal deaths was associated with preventable and treatable conditions. Education on neonatal resuscitation and postnatal management, and the introduction of an on-call doctor for high-risk deliveries might have contributed to the reduction in neonatal mortality over time.

Keywords: Low-resource setting, Mortality, Neonates, Risk factors

* Correspondence: daniele.trevisanuto@unipd.it

${ }^{6}$ Department of Woman's and Child's Health, University of Padua, Via Giustiniani 3, 35128 Padua, Italy

Full list of author information is available at the end of the article

C The Author(s). 2020 Open Access This article is licensed under a Creative Commons Attribution 4.0 International License, which permits use, sharing, adaptation, distribution and reproduction in any medium or format, as long as you give appropriate credit to the original author(s) and the source, provide a link to the Creative Commons licence, and indicate if changes were made. The images or other third party material in this article are included in the article's Creative Commons licence, unless indicated otherwise in a credit line to the material. If material is not included in the article's Creative Commons licence and your intended use is not permitted by statutory regulation or exceeds the permitted use, you will need to obtain permission directly from the copyright holder. To view a copy of this licence, visit http://creativecommons.org/licenses/by/4.0/. The Creative Commons Public Domain Dedication waiver (http://creativecommons.org/publicdomain/zero/1.0/) applies to the data made available in this article, unless otherwise stated in a credit line to the data. 


\section{Background}

Although under-5 mortality has decreased in the last two decades, neonatal mortality remains a global health challenge [1]. Worldwide, 2.4 million neonates die every year, a third of them within the first day of life $[1,2]$. The vast majority of neonatal deaths occur in sub-Saharan Africa and Southern Asia [3, 4]. The main causes of neonatal mortality are prematurity (35\%), intrapartum-related events (24\%) and infections (15\%) [5].

Despite achieving notable progress in neonatal mortality during 1999-2018 (from 49.5 deaths to 28.1 deaths per 1000 live births), Ethiopia has still one of the highest neonatal mortality rates worldwide [6]. Infant mortality is considered a standard indicator for the assessment of a country health status [7] and warrants for continuous research on interventions to achieve the United Nations Sustainable Development Goals (SDGs) [8]. Many neonatal deaths can be prevented with feasible and low-cost interventions [9]. Knowledge of risk factors for mortality can be useful in identifying areas of intervention and planning appropriate strategies to improve neonatal prognosis [10].

Previous studies showed varying figures (neonatal mortality $5.7-16.5 \%$ ) and heterogeneous risk factors for neonatal mortality (i.e. lack of antenatal care, outborn infants, home delivery, cesarean section, multiple birth, low birth weight, inability at cry at birth, perinatal asphyxia, need for resuscitation at birth, and respiratory distress syndrome) across different areas of Ethiopia [11-13], thus requiring further investigation to expand the knowledge and support the identification of appropriate interventions to reduce neonatal mortality [14]. This study aimed to assess the risk factors for mortality among neonates admitted to a special care unit in a referral hospital in rural Ethiopia.

\section{Methods}

\section{Study design}

This was a retrospective observational study on risk factors for mortality among neonates admitted to the special care unit of the St. Luke Wolisso Hospital (Ethiopia) from January 2014 to December 2017. The study was conducted according to Helsinki Declaration principles, and was approved by the Ethical Review Committee of St Luke Catholic Hospital and College of Nursing and Midwifery (ref. 245/2020), which waived the need for patient written consents, given the retrospective nature of the study and the use of anonymized data.

\section{Setting}

The study was conducted at the special care unit of the St. Luke Wolisso Hospital (Ethiopia), where around 3500 deliveries occur every year. This is a referral, private, nonprofit hospital located in Wolisso town, which is the capital of the Southwest Shoa Zone in the Oromiya region. The area has a population of about 1.1 million inhabitants and is served by 81 health facilities (including only one hospital). At St. Luke Wolisso Hospital, midwives were responsible for maternal and neonatal management at delivery. Medical transport system was not available in the area and outborn infants were brought to the hospital by their families. The special care unit consisted in one room with 10 beds and was cared for by two daytime nurses and one nighttime nurse. Phototherapy, intravenous therapies and oxygen supplementation (but not equipment for respiratory support) were offered. Availability of pulse oxymeter was limited. During the study period, education on neonatal resuscitation (Helping Babies Breathe program) and courses on postnatal management were offered to midwives and nurses, and the presence of an on-call doctor for high-risk deliveries was introduced.

\section{Patients}

All neonates admitted to the special care unit at the St. Luke Wolisso Hospital between 2014 and 2017 were included in the study. There were no exclusion criteria.

\section{Data collection}

All data were retrospectively retrieved from hospital charts and entered in an anonymized dataset. Data included neonatal characteristics (i.e. age, sex, weight), admission information (neonatal temperature, outborn/ inborn, mode of delivery, main diagnoses), length of stay and outcome. Clinical definitions are summarized in Table 1 [15-21].

\section{Statistical analysis}

Continuous variables were expressed as median and interquartile range (IQR), and categorical variables as number and percentage. Association between categorical variables was evaluated with Chi Square test. A logistic regression model was applied to identify predictors of mortality among clinically relevant factors (year of admission, mode of delivery, birthplace, age and temperature at admission, sex, birth weight, early-onset sepsis, late-onset sepsis, intrapartum-related complications, meconium aspiration syndrome, respiratory distress, transient tachypnea of the newborn, other infections, malformations). Multicollinearity was assessed using variance inflation factor (VIF), with values $>4$ suggesting further investigation and values $>10$ indicating need for correction for multicollinearity. Estimated effects from the model were expressed as odds ratios (OR) with 95\% confidence intervals (CI). Trends over time of the prevalence of prognostic factors were investigated with linear and logistic regression models where time was modeled with linear and quadratic terms. All tests were 2 -sided and a $p$-value less than 0.05 was considered statistically significant. Statistical analysis was performed 
Table 1 Clinical definitions

\begin{tabular}{|c|c|}
\hline Diagnosis at admission & Definition \\
\hline Sepsis & $\begin{array}{l}\text { Maternal history of fever or prolonged rupture of membranes }>18 \mathrm{~h} \text {, and/or neurologic findings } \\
\text { (apnea, convulsions, unconsciousness), and/or moderate hypothermia }\left(<36^{\circ} \mathrm{C}\right) \text { or hyperthermia } \\
\left(>37.5^{\circ} \mathrm{C}\right) \text {, and/or breastfeeding difficulties }[15-18] \text { within } 72 \mathrm{~h} \text { (early onset) or later (late onset). }\end{array}$ \\
\hline Other infections & Skin infections, abscesses, genital-urinary infections, pneumonia. \\
\hline Low birthweight & $\begin{array}{l}\text { Birthweight below } 2500 \mathrm{~g} \text { [16]. } \\
\text { Sub-groups in the manuscript were: } \\
\text {-LBW (low birthweight, 1500-2499 g), } \\
\text {-VLBW (very low birth weight, 1000-1499 g), } \\
\text {-ELBW (extremely low birth weight, < } 1000 \mathrm{~g} \text { ). }\end{array}$ \\
\hline Intrapartum-related complications & $\begin{array}{l}\text { Failure to initiate spontaneous regular respirations after birth and/or } 5 \text {-min Apgar score less than } \\
7 \text { and/or Sarnat \& Sarnat stage } 2 \text { or more at admission }[10,15,19] \text {. }\end{array}$ \\
\hline Meconium aspiration syndrome (MAS) & Presence of respiratory distress in infants born through meconium-stained amniotic fluid [20]. \\
\hline Respiratory distress & $\begin{array}{l}\text { Signs of respiratory insufficiency (tachypnea, dyspnea, and grunting) and need for oxygen } \\
\text { supplementation for more than } 2 \text { days [21]. }\end{array}$ \\
\hline Transient tachypnea of the newborn & Presence of tachypnea and need for oxygen supplementation within 2 days of life [21]. \\
\hline Malformations & $\begin{array}{l}\text { Diagnosis was based on clinical examination and/or radiological investigation (i.e. X-rays and } \\
\text { Doppler ultrasound). Magnetic resonance imaging (MRI), computer tomography (CT), } \\
\text { chromosome analysis and chromosome microarray were not available in the hospital. }\end{array}$ \\
\hline Neonatal hypothermia at admission & $\begin{array}{l}\text { Mild hypothermia: } 36-36.4^{\circ} \mathrm{C}[16] . \\
\text { Moderate hypothermia: neonatal temperature } 32-35.9^{\circ} \mathrm{C}[16] \\
\text { Severe hypothermia: neonatal temperature }<32^{\circ} \mathrm{C}[16]\end{array}$ \\
\hline Neonatal hyperthermia at admission & Neonatal temperature $>37.5^{\circ} \mathrm{C}[16]$. \\
\hline
\end{tabular}

using R 3.5 software (R Foundation for Statistical Computing, Vienna, Austria) [22].

\section{Results \\ Patients}

Overall, 4182 neonates (2424 males and 1758 females) were admitted to the special care unit of the St. Luke Wolisso Hospital (Ethiopia) from January 2014 to December 2017. Neonatal characteristics are reported in Table 2. Median age at admission was 1 day (IQR 1-4). Almost half of neonates were outborn (1577/3521, $44.8 \%$ ), while the information was not available in 661 neonates. Among 1179 inborn neonates who were admitted at day of birth, 379 (32\%) had moderate hypothermia $\left(32-36^{\circ} \mathrm{C}\right)$ and none severe hypothermia $\left(<32{ }^{\circ} \mathrm{C}\right)$. Moderate hypothermia was not different $(p=$ 0.75 ) in neonates born through vaginal delivery (439/ $1794,26.9 \%)$ and in those born through caesarean section (109/480, 28.6\%). Moderate hypothermia was $24.8 \%$ $(323 / 1300)$ in outborn and $28.3 \%(442 / 1560)$ in inborn neonates $(p=0.04)$.

\section{Diagnosis at admission}

The most common diagnoses at admission were birthweight $<2500 \mathrm{~g}(1,426,34.1 \%)$, early-onset sepsis (787, $18.8 \%)$, respiratory distress $(644,15.4 \%)$, intrapartumrelated complications $(595,14.2 \%)$ and late-onset sepsis $(540,12.9 \%)$ (Table 3). Median length of stay was 5 days (IQR 4-8). Eight neonates with spina bifida were transferred to another center for surgical treatment.

\section{Neonatal mortality}

Proportion of deaths was 17\% (709/4182) and was highest in ELBW and VLBW neonates (27/31, 87.1\%, and $137 / 270,50.7 \%$, respectively), and in those with intrapartum-related complications (173/595, 29.1\%), malformation $(36 / 129,27.9 \%)$ or respiratory distress $(146 / 644,22.7 \%)$ (Table 3). The majority of deaths $(84 \%)$ occurred during the first week of admission.

\section{Predictors of neonatal mortality}

At multivariable analysis (Table 4), increased likelihood of mortality was associated with outborn neonates (OR 1.64, 95\% CI 1.31 to 2.06 ) and with diagnosis of lateonset sepsis (OR 1.63, 95\% CI 1.12 to 2.36), LBW (OR 2.48, 95\% CI 2.00 to 3.09), VLBW (OR 11.71, 95\% CI 8.63 to 15.94), ELBW (OR 76.04, 95\% CI 28.54 to 263.82), intrapartum-related complications (OR 4.69, 95\% CI 3.55 to 6.20 ), MAS (2.34, 95\% CI 1.15 to 4.43 ), respiratory distress (OR 2.25, 95\% CI 1.72 to 2.95), other infections (OR 1.92, 95\% CI 1.31 to 2.81) or malformations (OR 2.32 95\% CI 1.49 to 3.57). On the other hand, being admitted in 2017 vs. 2014 (OR 0.71, 95\% CI 0.52 to 0.97 ) and older age at admission (OR $0.95,95 \% \mathrm{CI}$ 0.93 to 0.97 ) were associated with decreased likelihood of mortality. Multicollinearity was not present (VIF $<2$ for all factors).

In the subsample of 1994 neonates with complete data on temperature at admission and mode of delivery, moderate hypothermia $\left(32-35.9^{\circ} \mathrm{C}\right)$ at admission was a predictor of mortality (OR 1.53, 95\% CI 1.09 to $2.15 ; p=$ 
Table 2 Neonatal characteristics at admission

\begin{tabular}{|c|c|c|c|}
\hline & All neonates & Neonates who survived & Neonates who died \\
\hline No. of neonates & 4182 & 3473 & 709 \\
\hline \multicolumn{4}{|l|}{ Year of admission: } \\
\hline 2014 & $692(16.5)$ & $563(16.2)$ & $129(18.2)$ \\
\hline 2015 & $1113(26.6)$ & $916(26.4)$ & $197(27.8)$ \\
\hline 2016 & $1278(30.6)$ & $1059(30.5)$ & 219 (30.9) \\
\hline 2017 & $1099(26.3)$ & $935(26.9)$ & $164(23.1)$ \\
\hline Age at admission, days ${ }^{a}$ & $1(1-4)$ & $1(1-5)$ & $1-(1,2)$ \\
\hline \multicolumn{4}{|l|}{ Sex: } \\
\hline Male & $2424(58.0)$ & $2006(57.8)$ & $418(59.0)$ \\
\hline Female & $1758(42.0)$ & $1467(42.2)$ & $291(41.0)$ \\
\hline \multicolumn{4}{|l|}{ Birth weight: } \\
\hline Normal weight ( $\geq 2500 \mathrm{~g}$ ) & $2756(65.9)$ & $2440(70.3)$ & $316(44.6)$ \\
\hline Low birth weight (1500-2499 g) & $1125(26.9)$ & $896(25.8)$ & $229(32.3)$ \\
\hline Very low birth weight (1000-1499 g) & $270(6.5)$ & $133(3.8)$ & $137(19.3)$ \\
\hline Extremely low birth weight $(<1000 \mathrm{~g})$ & $31(0.7)$ & $4(0.1)$ & $27(3.8)$ \\
\hline \multicolumn{4}{|l|}{ Temperature at admission: ${ }^{b}$} \\
\hline$<32^{\circ} \mathrm{C}$ & $0(0.0)$ & $(0.0)$ & $0(0.0)$ \\
\hline $32-35.9^{\circ} \mathrm{C}$ & $779(26.7)$ & $583(24.0)$ & $196(40.5)$ \\
\hline $36-36.4^{\circ} \mathrm{C}$ & $759(26.1)$ & $634(26.1)$ & $125(25.8)$ \\
\hline $36.5-37.5^{\circ} \mathrm{C}$ & $948(32.5)$ & $822(33.8)$ & $126(26.0)$ \\
\hline$>37.5^{\circ} \mathrm{C}$ & $428(14.7)$ & $391(16.1)$ & $37(7.7)$ \\
\hline Outborn ${ }^{c}$ & $1577(44.8)$ & $1291(44.3)$ & $286(47.0)$ \\
\hline \multicolumn{4}{|l|}{ Mode of delivery: ${ }^{d}$} \\
\hline Vaginal & $1794(73.1)$ & $1422(71.1)$ & $372(82.1)$ \\
\hline Instrumental & $179(7.3)$ & $158(7.9)$ & $21(4.7)$ \\
\hline Caesarean section & $480(19.6)$ & $420(21.0)$ & $60(13.2)$ \\
\hline
\end{tabular}

Data expressed as No. (\%) or ${ }^{\text {a }}$ median (IQR). Data not available in ${ }^{\mathrm{b}} 1268,{ }^{\mathrm{c}} 661$ and ${ }^{\mathrm{d}} 1729$ neonates

$0.01)$, while mild hypothermia $\left(36-36.4^{\circ} \mathrm{C}\right)$, hyperthermia $\left(>37.5^{\circ} \mathrm{C}\right)$ and mode of delivery were not associated with mortality ( $p=0.71, p=0.98$ and $p=0.67$, respectively).

\section{Change over time in predictors of neonatal mortality}

During the study period, there was an increase of outborn neonates $(p=0.02)$ and those with late-onset sepsis $(p<0.0001)$, and a decrease of neonates with moderate hypothermia $(p=0.003)$ or other infections $(p=0.0003)$ (Table 5). The proportion of admissions for LBW ( $p=$ $0.0001)$ or malformations $(p=0.01)$ displayed a U-shaped trend, while the proportion of admissions for intrapartumrelated complications $(p=0.04)$ or respiratory distress $(p<$ $0.0001)$ displayed an inverted U-shaped trend (Table 5).

\section{Discussion}

Our findings indicated some neonatal characteristics (outborn, lower weight and age at admission) and a subset of diagnoses (hypothermia at admission, late-onset sepsis, low birth weight, intrapartum-related complications, MAS, respiratory distress, malformations and other infections) as risk factors for mortality after admission to the special care unit of a low-resource setting.

While neonatal mortality in high-resource countries is usually due to unpreventable causes, the majority of neonatal deaths in low-resource areas occur from preventable and treatable diseases, including intrapartum-related complications, prematurity and infections $[9,23]$.

In the last two decades, Ethiopia has succeeded in reducing neonatal mortality rate from 49.5 deaths to 28.1 deaths per 1000 live births thanks to many efforts from the government and other stakeholders [24]. Despite the implementation of the National Child Survival Strategy (2005-2015) [25], Ethiopia has still one of the highest neonatal mortality rates worldwide [6]. Investigation of mortality among neonates admitted to the special care unit in a low-resource setting is an important step for planning appropriate interventions $[9,23]$.

The magnitude of neonatal mortality was $17 \%$ among admissions to the special care unit of the St. Luke 
Table 3 Main diagnoses at admission and mortality rate according to diagnosis at admission

\begin{tabular}{lll}
\hline & No. of neonates & Deaths \\
\hline Early-onset sepsis & $787(18.8)$ & $107 / 787(13.6)$ \\
Late-onset sepsis & $540(12.9)$ & $75 / 540(13.9)$ \\
Low birth weight $(1500-2499 \mathrm{~g})$ & $1125(26.9)$ & $229 / 1125(20.4)$ \\
Very low birth weight $(1000-1499 \mathrm{~g})$ & $270(6.5)$ & $137 / 270(50.7)$ \\
Extremely low birth weight $(<1000 \mathrm{~g})$ & $31(0.7)$ & $27 / 31(87.1)$ \\
Intrapartum-related complications & $595(14.2)$ & $173 / 595(29.1)$ \\
Meconium aspiration syndrome & $67(1.6)$ & $12 / 67(17.9)$ \\
Respiratory distress & $644(15.4)$ & $146 / 644(22.7)$ \\
Transient tachypnea of the newborn & $129(3.1)$ & $8 / 129(6.2)$ \\
Other infections & $490(11.7)$ & $62 / 490(12.7)$ \\
Malformations & $129(3.1)$ & $36 / 129(27.9)$ \\
Hypothermia/hyperthermia: ${ }^{a}$ & & Nil \\
$\quad$ Severe hypothermia $\left(<32^{\circ} \mathrm{C}\right)$ & $0(0.0)$ & $125 / 759(16.5)$ \\
$\quad$ Moderate hypothermia $\left(32-36^{\circ} \mathrm{C}\right)$ & $779(26.7)$ & $196 / 779(25.2)$ \\
$\quad$ Mild hypothermia $\left(36-36.4^{\circ} \mathrm{C}\right)$ & $759(26.1)$ & $37 / 428(8.6)$ \\
$\quad$ Hyperthermia $\left(>37.5^{\circ} \mathrm{C}\right)$ & $428(14.7)$ &
\end{tabular}

Wolisso Hospital during 2014-2017, which laid in the mortality range of previous studies in Ethiopia [11-13, $26]$. The majority of deaths (84\%) occurred during the first week of admission - in agreement with previous studies [12, 26] - with low birth weight, intrapartumrelated complications, malformation and respiratory distress representing a heavy burden on neonatal mortality. These findings suggest the need for further efforts in improving labour, intrapartum and immediate postnatal newborn care practices [11].

Our analysis of risk factors of mortality confirmed the role of low birth weight [9], which is known to contribute to the largest number of both admissions and deaths in low-resource settings. Thermal care and appropriate feeding play an important role in these neonates, thus prevention and treatment of hypothermia (i.e. kangaroo mother care) and the promotion of early and exclusive breastfeeding are warranted [27]. Despite the very high mortality among ELBW and VLBW infants, their limited occurrence along with constrains for their treatment in low-resource settings suggested that efforts should target neonates with birth weight $1500-2500 \mathrm{~g}$. In addition, other comorbidities (intrapartum-related complications, late-onset sepsis, MAS, respiratory distress) and malformations were also associated with increased risk of neonatal mortality. All these factors can be both preventable (through appropriate antenatal and perinatal care) and cared for (with available skills and equipment) in the special care unit [9]. Quality improvement initiatives to reduce neonatal mortality should focus on strengthening the continuum of care including fetal, intrapartum and postnatal phases [9].

Outborn neonates and those with moderate hypothermia at admission were also identified as subjects at high risk of mortality. This is noteworthy since half of admissions were outborn, which mirrors the geographical distribution of population in Ethiopia, where over $80 \%$ of people resides in the rural part of the country [24]. Of note, our data suggested that the increased mortality risk in outborn neonates was not due to their temperature at admission.

About half of neonates were hypothermic at admission, thus underlying the importance of thermal control during the postnatal period [28]. Neonatal hypothermia is common in both health facilities and homes, even in tropical environments. While hypothermia is not often considered a direct cause of death, it contributes to a substantial proportion of neonatal mortality, mostly as a comorbidity of severe neonatal infections, preterm birth, and intrapartum-related complications [28, 29]. Of note, the surprising high proportion of hypothermia among inborn neonates calls for urgent actions for preventing thermal losses immediately after delivery. In lowresource settings, such condition is likely to persist in the days following birth, with negative impact on prognosis [30].

During the study period, the implementation of quality improvement interventions (education on neonatal resuscitation, courses on postnatal management, and the introduction of an on-call doctor for high-risk deliveries) 
Table 4 Multivariable analysis of predictors of mortality

\begin{tabular}{|c|c|c|}
\hline & p-value & Odds ratio ( $95 \%$ confidence interval) \\
\hline Year of admission: & 0.03 & \\
\hline 2014 & & Reference \\
\hline 2015 & & $1.00(0.73$ to 1.36$)$ \\
\hline 2016 & & 0.86 (0.63 to 1.16$)$ \\
\hline 2017 & & $0.71(0.52$ to 0.97$)$ \\
\hline Age at admission, days & 0.0003 & 0.95 (0.93 to 0.97$)$ \\
\hline Sex: & 0.62 & \\
\hline Female & & Reference \\
\hline Male & & $1.05(0.86$ to 1.28$)$ \\
\hline Birth weight: & $<0.0001$ & \\
\hline Normal weight ( $\geq 2500 \mathrm{~g}$ ) & & Reference \\
\hline Low birth weight (1500-2499 g) & & 2.48 (2.00 to 3.09$)$ \\
\hline Very low birth weight (1000-1499 g) & & 11.71 (8.63 to 15.94$)$ \\
\hline Extremely low birth weight $(<1000 \mathrm{~g})$ & & 76.04 (28.54 to 263.82 ) \\
\hline Birthplace: & $<0.0001$ & \\
\hline Inborn & & Reference \\
\hline Outborn & & 1.64 (1.31 to 2.06$)$ \\
\hline Early-onset sepsis: & 0.96 & \\
\hline No & & Reference \\
\hline Yes & & $0.99(0.75$ to 1.31$)$ \\
\hline Late-onset sepsis: & 0.01 & \\
\hline No & & Reference \\
\hline Yes & & 1.63 (1.12 to 2.36$)$ \\
\hline Intrapartum-related complications: & $<0.0001$ & \\
\hline No & & Reference \\
\hline Yes & & 4.69 (3.55 to 6.20$)$ \\
\hline Meconium aspiration syndrome: & 0.01 & \\
\hline No & & Reference \\
\hline Yes & & 2.34 (1.15 to 4.43$)$ \\
\hline Respiratory distress: & $<0.0001$ & \\
\hline No & & Reference \\
\hline Yes & & $2.25(1.72$ to 2.95$)$ \\
\hline Transient tachypnea of the newborn: & 0.26 & \\
\hline No & & Reference \\
\hline Yes & & $0.62(0.25$ to 1.33$)$ \\
\hline Other infections: & 0.0007 & \\
\hline No & & Reference \\
\hline Yes & & $1.93(1.31$ to 2.81$)$ \\
\hline Malformations: & 0.0002 & \\
\hline No & & Reference \\
\hline Yes & & $2.32(1.49$ to 3.57$)$ \\
\hline
\end{tabular}

In the subsample of 1994 neonates with complete data on temperature at admission and mode of delivery, moderate hypothermia $\left(32-35.9^{\circ} \mathrm{C}\right)$ at admission was a predictor of mortality $(\mathrm{OR} 1.53,95 \% \mathrm{Cl} 1.09$ to $2.15 ; \mathrm{p}=0.01)$, while mild hypothermia $\left(36-36.4^{\circ} \mathrm{C}\right)$, hyperthermia $\left(>37.5^{\circ} \mathrm{C}\right)$ and mode of delivery were not associated with mortality $(p=0.71, p=0.98$ and $p=0.67$, respectively) 
Table 5 Summary of predictors of mortality during the study period

\begin{tabular}{|c|c|c|c|c|c|}
\hline & 2014 & 2015 & 2016 & 2017 & $p$-value \\
\hline No. of neonates & 692 & 1113 & 1278 & 1099 & - \\
\hline Age at admission, days ${ }^{a}$ & $1(1-4)$ & $1(1-4)$ & $1(1-5)$ & $1(1-4)$ & 0.77 \\
\hline Low birth weight (1500-2499 g) & $202(29.1)$ & $258(23.2)$ & $323(25.3)$ & $342(31.1)$ & $0.0001^{b}$ \\
\hline Very low birth weight (1000-1499 g) & $46(6.6)$ & $56(5.0)$ & $82(6.4)$ & $86(7.8)$ & 0.09 \\
\hline Extremely low birth weight $(<1000 \mathrm{~g})$ & $3(0.4)$ & $9(0.8)$ & $11(0.9)$ & $8(0.7)$ & 0.56 \\
\hline Outborn & $202(39.1)$ & $401(45.6)$ & $514(46.6)$ & $460(45.1)$ & $0.02^{b}$ \\
\hline Moderate hypothermia $\left(32-35.9^{\circ} \mathrm{C}\right)$ & $171(38.4)$ & $156(21.6)$ & $218(28.2)$ & $234(24.0)$ & $0.003^{b}$ \\
\hline Late-onset sepsis & $56(8.1)$ & $127(11.4)$ & $168(13.1)$ & $189(17.2)$ & $<0.0001$ \\
\hline Intrapartum-related complications & $84(12.1)$ & $163(14.6)$ & 199 (15.6) & 149 (13.6) & $0.04^{b}$ \\
\hline Meconium aspiration syndrome & $5(0.7)$ & $18(1.6)$ & $24(1.9)$ & $20(1.8)$ & 0.09 \\
\hline Respiratory distress & $67(9.7)$ & 177 (15.9) & 302 (23.6) & $98(8.9)$ & $<0.0001^{b}$ \\
\hline Other infections & 87 (12.6) & $172(15.4)$ & $127(9.9)$ & $104(9.5)$ & 0.0003 \\
\hline Malformations & $32(4.6)$ & $23(2.1)$ & $37(2.9)$ & 37 (3.4) & $0.01^{b}$ \\
\hline
\end{tabular}

Data expressed as $\mathrm{n}(\%)$ or ${ }^{\mathrm{a}}$ median (IQR). ${ }^{\mathrm{b}}$ Quadratic curve over time

could contribute to explain the mortality reduction in 2014-2017 [31]. In addition, we observed different trends over time of factors associated with neonatal mortality. While these data provide useful information about the changing characteristics of admitted neonates, the interpretation goes beyond the scope of the present study and should rely on a longer time span.

During the study, problems in documentation emerged when retrieving data from hospital charts. In fact, information on important prognostic indicators (such as temperature at admission and place/mode of delivery) was missing in $15-30 \%$ of the records. The underreporting of such indicators highlights the need for enhancing the awareness of the importance of including those measurements among routine care [32].

This study has some limitations. First, it is a singlecenter study thus generalizability is limited to similar settings. Second, the retrospective data collection limited the quality and completeness of available information. Third, the diagnosis was mostly based on clinical examination due to the limited availability of laboratory and instrumental equipment.

Our study adds information about risk for mortality among neonates admitted to a special care unit in Ethiopia, where available literature on risk factors for neonatal mortality is limited [11-13, 27]. Our findings confirm that the majority of neonatal deaths seemed to be associated with preventable and treatable conditions [14]. Thus, improvements of referral system, ante- and perinatal care, and postnatal management are warranted to reduce neonatal mortality $[11,15]$. Of note, education to health care givers, audits and continuous feedback should be implemented to improve quality and completeness of documentation.
Our findings also contribute to feed up an on-going systematic review which aims at filling the gap in understanding burden and risk factors of neonatal mortality in Ethiopia [14]. The summary of the available evidence will inform health policy makers and stakeholders about which factors should be targeted to reduce neonatal mortality in Ethiopia [14].

\section{Conclusions}

Our findings showed that neonatal mortality was associated with admission at early age, low birthweight, being outborn, late-onset sepsis, intrapartum-related complications, meconium aspiration syndrome, respiratory distress, infections, malformations and hypothermia. Education on neonatal resuscitation and postnatal management, and the introduction of an on-call doctor for high-risk deliveries might have contributed to the reduction in neonatal mortality over time.

\section{Abbreviations \\ Cl: Confidence interval; ELBW: Extremely low birthweight; LBW: Low birthweight; MAS: Meconium aspiration syndrome; OR: Odds ratio; VLBW: Very low birthweight}

\section{Acknowledgements}

Not Applicable.

\section{Authors' contributions}

FC participated in the conception and design of the study, performed the data analysis, wrote the initial draft of the manuscript and gave a substantial contribution to the design and interpretation of the data. TB and DAY contributed to data collection, strictly coordinated all the local phases of the study and made a substantial contribution to the interpretation of the data. FM and GP participated in the conception and design of the study and made a substantial contribution to the analysis and interpretation of the data. DT conceived and designed the study; made substantial contribution to the analysis and interpretation of the data; redrafted the manuscript and revised it for important intellectual content. All authors contributed to the 
final version of the manuscript and provided critical interpretation of the contents and approved the manuscript for publication.

\section{Funding}

No funding was secured for this study.

\section{Availability of data and materials}

The material of the current study is be available from the corresponding author on reasonable request.

\section{Ethics approval and consent to participate}

The study was approved by the Ethical Review Committee of St Luke Catholic Hospital and College of Nursing and Midwifery (ref. 245/2020), which waived the need for patient written consents, given the retrospective nature of the study and the use of anonymized data.

\section{Consent for publication}

Not applicable.

\section{Competing interests}

The authors have no conflicts of interest to disclose.

\section{Author details}

${ }^{1}$ Independent statistician, Solagna, Italy. ${ }^{2}$ Doctors with Africa CUAMM Wolisso, Ethiopia. ${ }^{3}$ Department of Neonatal and Pediatric Critical Care, University of Verona, Verona, Italy. ${ }^{4}$ Public Health Coordinator, St. Luke Catholic Hospital, Wolisso, Ethiopia. ${ }^{5}$ Doctors with Africa CUAMM, Padua, Italy. ${ }^{6}$ Department of Woman's and Child's Health, University of Padua, Via Giustiniani 3, 35128 Padua, Italy.

Received: 15 May 2020 Accepted: 13 November 2020

Published online: 23 November 2020

\section{References}

1. Burstein R, Henry NJ, Collison ML, Marczak LB, Sligar A, Watson S, et al. Mapping 123 million neonatal, infant and child deaths between 2000 and 2017. Nature. 2019:574:353-8.

2. World Health Organization, 2020. Newborns: improving survival and wellbeing. https://www.who.int/news-room/fact-sheets/detail/newbornsreducing-mortality (accessed 26 October 2020).

3. Lawn JE, Cousens S, Zupan J. 4 million neonatal deaths: when? Where? Why? Lancet. 2005:365(9462):891-900.

4. United Nations Intern-agency Group for Child Mortality Estimation. Global Database - TARGET 3.2. 2018. Available from: https://unstats.un.org/sdgs/ indicators/database/

5. Ehret DY, Patterson JK, Bose CL. Improving neonatal care: a global perspective. Clin Perinatol. 2017 Sep;44(3):567-82.

6. World Data Atlas. World and regional statistics, national data, maps, rankings. https://knoema.com/atlas/Ethiopia/Neonatal-mortality-rate. Accessed 18 Nov 2020.

7. Gonzalez RM, Gilleskie D. Infant mortality rate as a measure of a Country's health: a robust method to improve reliability and comparability. Demography. 2017:54(2):701-20.

8. United Nations. The sustainable development goals. https://www.un.org/ sustainabledevelopment. Accessed 18 Nov 2020.

9. Bhutta ZA, Das JK, Bahl R, Lawn JE, Salam RA, Paul VK, Sankar MJ, Blencowe H, Rizvi A, Chou VB, Walker N. Lancet newborn interventions review group; lancet every newborn study group. Can available interventions end preventable deaths in mothers, newborn babies, and stillbirths, and at what cost? Lancet. 2014;384:347-70.

10. Ersdal HL, Mduma E, Svensen E, Perlman J. Birth asphyxia: a major cause of early neonatal mortality in a Tanzanian rural hospital. Pediatrics. 2012;129: e1238-43.

11. Roro EM, Tumtu MI, Gebre DS. Predictors, causes, and trends of neonata mortality at Nekemte Referral Hospital, east Wollega Zone, western Ethiopia (2010-2014). Retrospective cohort study. PLoS One. 2019;14(10):e0221513.

12. Elmi Farah A, Abbas AH, Tahir AA. Trends of admission and predictors of neonatal mortality: a hospital based retrospective cohort study in Somali region of Ethiopia. PLoS One. 2018;13(9):e0203314
13. Orsido TT, Asseffa NA, Berheto TM. Predictors of neonatal mortality in neonatal intensive care unit at referral Hospital in Southern Ethiopia: a retrospective cohort study. BMC Pregnancy Childbirth. 2019;19(1):83

14. Tekelab T, Akibu M, Tagesse N, Tilhaun T, Yohanes Y, Nepal S. Neonatal mortality in Ethiopia: a protocol for systematic review and meta-analysis. Syst Rev. 2019;8(1):103.

15. Cavicchiolo ME, Lanzoni P, Wingi MO, Pizzol D, Daverio M, Da Dalt L, Putoto $G$, Trevisanuto D. Reduced neonatal mortality in a regional hospital in Mozambique linked to a quality improvement intervention. BMC Pregnancy Childbirth. 2016;16:366.

16. World Health Organization. Managing newborn problems: a guide for doctors, nurses, and midwives. Geneva: World Health Organization; 2003. https://apps.who.int/iris/handle/10665/42753.

17. Young Infants Clinical Signs Study Group. Clinical signs that predict severe illness in children under age 2 months: a multicentre study. Lancet. 2008; 371:135-42.

18. Seale AC, Obiero CW, Berkley JA. Rational development of guidelines for management of neonatal sepsis in developing countries. Curr Opin Infect Dis. 2015;28:225-30.

19. Sarnat HB, Sarnat MS. Neonatal encephalopathy following fetal distress. A clinical and electroencephalographic study. Arch Neurol. 1976;33:696-705

20. Bhat RY, Rao A. Meconium-stained amniotic fluid and meconium aspiration syndrome: a prospective study. Ann Trop Paediatr. 2008;28:199-203.

21. Stark AR, Hansen AR, Martin CR, Eichenwald EC. Cloherty and Stark's Manual of Neonatal Care. Lippincott Williams and Wilkins. 2017.

22. Core Team R. R: a language and environment for statistical computing. In: R Foundation for statistical computing, Vienna, Austria; 2018. URL https:// www.R-project.org/.

23. Chow S, Chow R, Popovic M, Lam M, Popovic M, Merrick J, Stashefsky Margalit RN, Lam H, Milakovic M, Chow E, Popovic J. A selected review of the mortality rates of neonatal intensive care units. Front Public Health. 2015;3:225.

24. UNICEF. National Newborn and Child Survival Strategy Document Brief Summary 2015/16-2019/20. https://www.unicef.org/ethiopia/media/391/file/ Child\%20Survival\%20Strategy\%20in\%20Ethiopia\%20.pdf. Accessed 18 Nov 2020

25. Federal Ministry of Health. National strategy for newborn and child survival in Ethiopia, 2015/16-2029/20 Addis Ababa, Ethiopia 2015.

26. Debelew GT, Afework MF, Yalew AW. Determinants and causes of neonatal mortality in Jimma zone, Southwest Ethiopia: A Multilevel Analysis of Prospective Follow-Up Study. PLoS One. 2014;9:e107184.

27. Conde-Agudelo A, Díaz-Rossello JL. Kangaroo mother care to reduce morbidity and mortality in low birthweight infants. Cochrane Database Syst Rev. 2016:2016(8):CD002771.

28. Trevisanuto D, Testoni D, de Almeida MFB. Maintaining normothermia: why and how? Semin Fetal Neonatal Med. 2018;23(5):333-9.

29. Lunze K, Bloom DE, Jamison DT, Hamer DH. The global burden of neonatal hypothermia: systematic review of a major challenge for newborn survival. BMC Med. 2013;11:24

30. Cavallin F, Calgaro S, Brugnolaro V, AHA S, Muhelo AR, Da Dalt L, Putoto G, Trevisanuto D. Impact of temperature change from admission to day one on neonatal mortality in a low-resource setting. BMC Pregnancy Childbirth. 2020;20(1):646.

31. Versantvoort JMD, Kleinhout MY, Ockhuijsen HDL, Bloemenkamp K, de Vries $W B$, van den Hoogen A. Helping babies breathe and its effects on intrapartum-related stillbirths and neonatal mortality in low-resource settings: a systematic review. Arch Dis Child. 2020;105(2):127-33.

32. Lawn JE, Blencowe H, Oza S, You D, Lee AC, Waiswa P, Lalli M, Bhutta Z, Barros AJ, Christian P, Mathers C. Cousens SN; lancet every newborn study group. Every newborn: progress, priorities, and potential beyond survival. ancet. 2014:384(9938):189-205.

\section{Publisher's Note}

Springer Nature remains neutral with regard to jurisdictional claims in published maps and institutional affiliations. 